\title{
A Design of Chaotic Cryptosystem Based on Linear Controller Parameterization
}

\author{
Takami Matsuo \\ Department of Human Welfare Engineering, Oita University \\ 700 Dannoharu, Oita, Japan, 870-1192 \\ matsuo@cc.oita-u.ac.jp
}

Keywords: Chua circuit, encrypter, decrypter, synchronization, linear fractional transformation, Youla parameterization

\begin{abstract}
A general methodology for designing chaotic and hyperchaotic cryptosystems was developed using the control systems theory. Grassi proposed a nonlinearobserver-based decrypter for the state of the encrypter. If we can design the decrypter without the knowledge of the parameters of the encrypter, the chaos-based secure communication systems are not secure. In this paper, we propose an observer-based chaotic communication system, which allows us to assign the relative degree and the zeros of its encrypter system. Moreover, we design a robust synchronizer based on linear controller parameterization.
\end{abstract}

\section{Introduction}

A general methodology for designing chaotic and hyperchaotic cryptosystems was developed using the control systems theory $[4,5,6,7]$. The chaotic communication system is closely related to the concept of chaos synchronization. Yang et al. proposed a new chaos-based secure communication scheme in an attempt to thwart the attacks proposed recently $[4,5]$. They have combined both conventional cryptographic method and synchronization of chaotic systems. Their cryptographic method consists of an encryption function (the multi-shift cipher), a decryption function (the inverse of the encryption function), a chaotic encrypter that generates the key signal for the encryption function, and a decrypter that estimates the key signal. The approach has a limitation since the crypto system design may fail if different chaotic circuits are utilized. From the control theoretic perspective, the transmitter and the receiver in the chaotic communication system can be considered as the nonlinear plant and its observer, respectively. Grassi et al. proposed a nonlinearobserver-based decrypter to reconstruct the state of the encrypter $[6,7]$. They extended the Chua's oscillator to the observer-based decrypter. The cryptosystem does not require initial conditions of the encrypter and the decrypter belonging to the same basin of attraction. If we can design a decrypter without the knowledge of the parameters of the encrypter, the chaos-based secure communication systems are not secure. Parameter identification and adaptive synchronization methods may be effective for intruders in building reconstruction mechanisms, even when a synchronizing system is not available. Therefore, it is important for secure issues to investigate whether adaptive identifiers without the system information of encrypter can be constructed or not. We have recently designed an observer-based chaotic communication system combining the cryptosystems proposed by Grassi et al. and by Liao et al. that allows us to assign the relative degree and the zeros of its encrypter system [14]. Especially, we constructed three cryptosystems based on a Chua's circuit by assigning its relative degree and zeros. The cryptosystem consists of an encryption function (the multi-shift cipher), a decryption function (the inverse of the encryption function), a chaotic encrypter that generates the key signal for the encryption function, and a decrypter that estimates the key signal. The proposed cryptosystem allows us to assign the relative degree and the zeros of the encrypter dynamics by selecting an output vector that generates a transmitted signal as partial states of the encrypter. Therefore, the encrypter dynamics should be design such that its relative degree is more than two and its zeros are unstable so as to fail to synchronize the cryptosystem.

In this paper, we add an observer-based chaotic communication system proposed by Grassi et al. to a dynamical compensator in its transmitted signal to improve the security of the cryptosystem. Specifically, we construct an output equation of the encrypter with a dynamics that stabilizes the error systems. The dynamics is designed via the linear controller parameterization to make a decrypter sensitive to modeling errors of the decrypter. We develop a design procedure of a free pa- 
rameter in the dynamics to improve the security of the cryptosystem using the robust control theory.

The following notation is used :

$$
\begin{array}{ll}
\mathcal{F}_{l}(G, Q) & : \text { lower LFT } \\
C(s I-A)^{-1} B+D & :=\left[\begin{array}{l|l}
A & B \\
\hline C & D
\end{array}\right]
\end{array}
$$

\section{Observer-Based Chaotic Communication System}

Grassi et al.[6] proposed a nonlinear-observer-based cryptosystem that is an extension of the cryptosystem proposed by Yang et al. The cryptographic method consists of an encryption function (the multi-shift cipher), a decryption function (the inverse of the encryption function), a chaotic encrypter that generates the key signal for the encryption function, and a decrypter that estimates the key signal. The transmitted signal through a public channel contains the nonlinear function that is equivalent to that of the encrypter. Liao et al.[7] presented another observer-based approach for chaotic secure communication systems. Their cryptosystem does not have an encryption function with a key signal and its transmitted signal is a sum of a linear function of the states of the encrypter and a plain text. In this paper, we present an observer-based chaotic communication system combining the cryptosystems proposed by Grassi et al. and Liao et al. which allows us to assign the relative degree and the zeros of its encrypter system. The proposed cryptosystem consists of four parts.

The cryptosystem consists of an encryption function (the multi-shift cipher), a decryption function (the inverse of the encryption function), a chaotic encrypter that generates the key signal for the encryption function, and a decrypter that estimates the key signal.

\section{- Part 1 : dynamic encrypter}

The chaotic encrypter is described by the following equations:

$$
\begin{aligned}
\dot{x} & =A x+b_{2} f(x)+b_{2} e_{n} \\
y_{0} & =c^{T} x \\
v & =P(s) y_{0} \\
y & =v+e_{n}+f(x)
\end{aligned}
$$

where $y$ is transmitted signal that includes the nonlinear function and $P(s)$ is a transfer function that lets a decrypter synchronize the encrypter. We call this transfer function a synchronizer.

- Part 2 : encryption function

Given a plaintext signal $p(t)$, the ciphertext $e_{n}(t)$ is given by

$$
e_{n}(t)=e_{n}(p(t), K(t))
$$

where $K(t)$ is a key signal that is generated by the encrypter dynamics and is given by the following equation:

$$
K(t)=k^{T} x
$$

The signal $e_{n}$ is a generic encryption function that makes use of the key signal and we choose a encryption function as the following $n$-shift cipher:

$$
\begin{aligned}
e_{n}(p(t), K(t)) & =q(\cdots q(q(p(t), K(t)), K(t)), \cdots), K(t)) \\
q(x, k) & = \begin{cases}(x+k)+2 h, & -2 h \leq(x+k) \leq-h \\
(x+k), & -h<(x+k)<h \\
(x+k)-2 h & h \leq(x+k) \leq 2 h\end{cases}
\end{aligned}
$$

- Part 3 : dynamic decrypter with free parameter

Given the encrypter, the decrypter is the following observer:

$$
\begin{aligned}
\dot{\hat{x}} & =A \hat{x}+b_{2} e_{y} \\
\hat{y}_{0} & =c^{T} \hat{x} \\
\hat{y} & =\hat{v}=P(s) \hat{y}_{0} \\
\hat{e}_{n} & =y-(\hat{y}+f(\hat{x})) \\
e_{y} & =y-\hat{y}=P(s)\left(y_{0}-\hat{y}_{0}\right)+e_{n}+f(x)
\end{aligned}
$$

where $\hat{e}_{n}$ is a recovered signal of the plain text.

\section{- Part 4: decryption function}

Using the estimated signals $\hat{K}(t)$ and $\hat{e}_{n}(t)$ by the decrypter, the estimate of the plaintext $\hat{p}(t)$ can be recovered by the following equations:

$$
\begin{aligned}
\hat{p}(t) & =d\left(\hat{e}_{n}(t), \hat{K}(t)\right) \\
\hat{K}(t) & =k^{T} \hat{x}
\end{aligned}
$$

where $\hat{K}$ is an estimate of the key signal and $d$ is the decryption function given by

$$
\left.\hat{p}(t)=q\left(\cdots q\left(q\left(\hat{e}_{n}(t),-\hat{K}(t)\right),-\hat{K}(t)\right), \cdots\right),-\hat{K}(t)\right) .
$$

Selecting an appropriate vector $c$, we can assign the zeros and relative degree of the transfer function $c^{T}(s I-$ $A)^{-1} b_{2}$ arbitrarily.

\section{Design of decrypter}

\subsection{Error equation and generalized system}

If the transmitted signal is disturbed by an additional disturbance $w(t)$, the signal is rewritten by

$$
y(t)=v(t)+e_{n}(t)+f(x(t))+w(t)
$$

Defining the estimation error $e(t)$ of the decrypter as $e(t)=\hat{x}(t)-x(t)$, we have the following error system:

$$
\begin{aligned}
\dot{e}(t) & =A e(t)-b_{2} \xi(t)+b_{2} w(t) \\
e_{K}(t) & =\hat{K}(t)-K(t)=k^{T} e(t) \\
e_{0}(t) & =c^{T} e(t) \\
\xi(t) & =P(s) e_{0}
\end{aligned}
$$


The recovered signal can be rewritten by

$$
\begin{aligned}
\hat{e}_{n} & =y-\hat{y}-f(\hat{x}) \\
& =v+e_{n}+f(x)+w-\hat{v}-f(\hat{x}) \\
& =e_{n}+(v-\hat{v})+(f(x)-f(\hat{x})) \\
& =e_{n}-P(s) c^{T} e+(f(x)-f(\hat{x}))
\end{aligned}
$$

Thus, if $\lim _{t \rightarrow \infty} w(t)=0$ and $\lim _{t \rightarrow \infty} e(t)=0$, then we attain the recover the plaintext, i.e. $\lim _{t \rightarrow \infty}\left(e_{n}(t)-\right.$ $\left.\hat{e}_{n}(t)\right)=0$. The generalized plant is defined as

$$
G(s)=\left[\begin{array}{c|cc}
A & -b_{2} & b_{2} \\
\hline k^{T} & 0 & 0 \\
c^{T} & 0 & 0
\end{array}\right]
$$

and the synchronizer $P(s)$ is a stabilizing compensator for the generalized plant $G(s)$. The design problem of the decrypter is summarized as follows:

Given a generalized plant $G(s)$, parameterize all synchronizer $P(s)$ that internally stabilize $G(s)$.

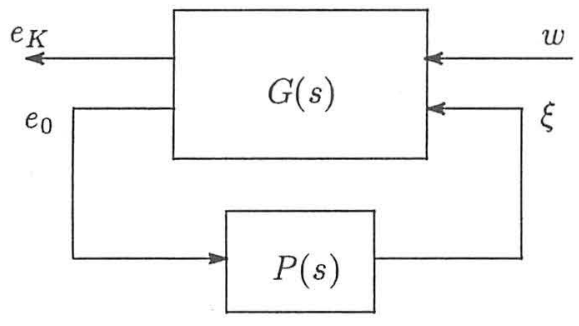

Figure 1: Generalized plant and synchronizer.

\subsection{Synchronizer parameterization}

When the generalized plant can be stabilized by a static output feedback, i.e. there exists an output feedback gain $k_{0}$ such that $A_{k}=A-b_{2} k_{0} c^{T}$ is stable, we can set $H_{0}=0, F_{0}=0$. In this case, the parameterization of all stabilizing compensators is as follows[13]:

$$
P(s)=\mathcal{F}_{l}\left(\tilde{P}_{c}(s), Q(s)\right)
$$

where $Q(s) \in R H_{\infty}$ and

$$
\tilde{P}_{c}(s)=\left[\begin{array}{c|cc}
A_{k} & 0 & b_{2} \\
\hline 0 & k_{0} & I \\
-c^{T} & I & 0
\end{array}\right] .
$$

We call this parameterization a synchronizer parameterization. By selecting $P(s)$ as constant gain $k_{0}$ i.e. $Q(s)=0$, the proposed cryptosystem is equivalent to that proposed by Grassi et al. Therefore, the free parameter $Q(s)$ can be designed to attain a high synchronization performance.

\section{3. $H_{\infty}$ synchronizer}

The transfer function $T_{e_{K} w}$ from the disturbance $w$ to the controlled signal $e_{k}$ is given by

$$
T_{e_{K} w}=\mathcal{F}_{l}(G, P)
$$

We define the following $H_{\infty}$ synchronization problem.

Given $\gamma>0$, find all admissible synchronizer $P(s)$, if there are any, such that $\left\|T_{e_{K} w}\right\|_{\infty}<\gamma$.

The proposed decrypter can recover the plain text by the transmitted signals when the generalized plant with synchronizer is internally stable. Moreover, the $H_{\infty}$ synchronizer has an additional synchronization property with respect to plant uncertainties.

\subsection{Design specification}

- Sensitivity for parametric uncertainty To attain a high security performance, the encrypter should be sensitive for parametric uncertainties, e.g. $A, b_{2}, k$, and $f(\cdot)$.

- Robustness for delay and noise in transmission line

To recover plain texts, he decrypter should be robust for noise and delay uncertainties in transmission line.

\section{Simulations}

We design a robust cryptosystem via Chua's circuit as in Yang et al.[4] and Fradkov et al.[11], and carry out simulations using MATLAB/Simulink.

\subsection{Encrypter based on Chua's circuit}

The chaotic encrypter based on the Chua's circuit is given by

$$
\begin{aligned}
\dot{x} & =A x+b_{2} f\left(x_{1}\right)+b_{2} e_{n} \\
y & =P(s) c^{T} x+e_{n}+f(x) \\
f\left(x_{1}\right) & =G_{b} x_{1}+\frac{1}{2}\left(G_{a}-G_{b}\right)\left(\left|x_{1}+1\right|-\left|x_{1}-1\right|\right) \\
A & =\left[\begin{array}{ccc}
-p_{1} & p_{1} & 0 \\
1 & -1 & 1 \\
0 & -p_{2} & -p_{3}
\end{array}\right] \\
b_{2} & =\left[\begin{array}{c}
-p_{1} \\
0 \\
0
\end{array}\right], x=\left[\begin{array}{l}
x_{1} \\
x_{2} \\
x_{3}
\end{array}\right]
\end{aligned}
$$

We select the parameters in the Chua's circuit given by Liao et al.[7] as $p_{1}=10, p_{2}=13.14, p_{3}=0.07727, G_{a}=$ -1.28 , and $G_{b}=-0.69$. The initial conditions are given by

$$
\begin{aligned}
& x_{1}(0)=1.1, x_{2}(0)=0, x_{3}(0)=0 \\
& \hat{x}_{1}(0)=0, \hat{x}_{2}(0)=0, \hat{x}_{3}(0)=0
\end{aligned}
$$


The encryption function is 30-shift cipher, the parameter $h$ is equal to 10 and the key signal $K(t)$ is the second state variable $x_{2}$, i.e.

$$
K(t)=\left[\begin{array}{lll}
0 & 1 & 0
\end{array}\right] x(t) .
$$

Throughout simulations, we use the plaintext signal as $p(t)=\sin 2 t$.

By assigning the vector $c^{T}$ such that $A-b_{2} c^{T}$ is stable, the proposed cryptosystem can assign its relative degree and zeros of the transfer function $J(p)=$ $c^{T}(p I-A)^{-1} b_{2}$ as in following three cases:

- Case 1 : Selecting the vector in the transmitted signal as

$$
c^{T}=-\left[\begin{array}{lll}
5 & 0 & 0
\end{array}\right],
$$

the relative degree, the zeros, and the poles of $J(p)$ are $1, \quad\{-0.5386 \pm j 3.5954\}$, and $\{-10.8998,-0.0887 \pm j 3.4709\}$ respectively. The eigenvalues of $A-b_{2} c^{T}$ are $\{-60.1684,-0.4544 \pm$ $j 3.6001\}$.

- Case 2 : Selecting the vector in the transmitted signal as

$$
c^{T}=-\left[\begin{array}{lll}
0 & 1 & 1
\end{array}\right],
$$

the relative degree and the zeros of $J(p)$ are 2 and $\{13.063\}$, respectively. The eigenvalues of $A-$ $b_{2} c^{T}$ are $\{-8.14275,-2.90182,-0.032702\}$.

By selecting $P(s)$ as constant gain $k_{0}$, the proposed cryptosystem is equivalent to that proposed by Grassi et al.. The Simulink configurations of the cryptosystem proposed by Grassi et al. and that proposed here are given by Figure 2 and 3, respectively.

\subsection{Nominal performance}

Figure 3 shows the Simulink configuration of the cryptosystem proposed in this paper. The vector $c^{T}$ in transmitted signal $y$ is selected as $c^{T}=-\left[\begin{array}{lll}5 & 0 & 0\end{array}\right]$. Figure 4 shows the transmitted signal and the plain text (above left), the cipher text (below left), the error vector between the encrypter and the decrypter (above right), and the estimation error of recovered signal (below right) when the synchronizer is selected as the constant gain $P(s)=k_{0}=5$ that is equivalent to the cryptosystem proposed by Grassi et al.. Figure 5 shows the same responses with an $H_{\infty}$ synchronizer. The generalized plant in designing the $H_{\infty}$ synchronizer is shown in Figure 6. Weighting function $W(s)$ in the exogenous signal is selected as $W(s)=\frac{2.1 L s}{L s+1}, L=0.001$ and $\gamma=1$. The $H_{\infty}$ synchronizer is obtained by using LMI toolbox.

\subsection{Robustness for time delay in transmission line}

Figure 7 shows the Simulink configuration of the cryptosystem with time delay in the transmission line.
Figures 8 and 9 show the responses of the Grassi-type decrypter and $H_{\infty}$-type decrypter in the presence of time delay $L=0.0001$ in transmission line, respectively.

\subsection{Robustness for noise in transmission line}

Figure 10 shows the Simulink configuration of the cryptosystem with noise in the transmission line. In this case, we use the low pass filter $\frac{1}{0.01 s+1}$ to get the recovered signal. The decrypter does not need a filter in synchronizing the encrypter. Figures 11 and 12 show the responses of the Grassi-type decrypter and $H_{\infty}$ type decrypter in the presence of Gaussian noise with the variance, 0.05 , in transmission line, respectively.

\section{Conclusion}

In this paper, we proposed a nonlinear observerbased chaotic communication system combining the cryptosystems proposed by Grassi et al. and Yang et al. The proposed chaotic system demonstrates good signal reconstruction abilities and robust performance for time delay in transmission line.

\section{References}

[1] Cuomo,K.M. and A.V. Oppenheim. "Synchronization of Lorenz-based chaotic circuits with applications to communications", IEEE Trans. CAS-I, 40, pp.626-633, 1993.

[2] Dedieu,H. and M.J Ogorzalek. "Identifiability and Identification of Chaotic Systems Based on Adaptive Synchronization", IEEE Trans. CAS-I, 44, pp.948-962, 1997.

[3] H. Nijmeijer and I.M. Mareels. "An Observer Looks at Synchronization", IEEE Trans. CAS-I, 44, pp.882-890, 1997.

[4] T. Yang, C.W. Wu and L.O. Chua. "Cryptography based on chaotic systems", IEEE Trans. CAS-I, 44, pp.469-472, 1997.

[5] T. Yang and L.O. Chua. "Impulsive control and synchronization of nonlinear dynamical systems and application to secure communication", Int. J. Bifurcation and Chaos, 7-3, pp.645-664, 1997.

[6] G. Grassi and S. Mascolo. "A System Theory Approach for Designing Cryptosystems Based on $\mathrm{Hy}$ perchaos", IEEE Trans. CAS-I, 46-9, pp.1135-1138, 1999.

[7] T.L. Liao and N.S. Huang. "An Observer-Based Approach for Chaotic Synchronization with Applications to Secure Communications", IEEE Trans. CAS-I, 46-9, pp.1144-1149, 1999.

[8] T.L. Liao and S.H. Lin. "Adaptive Control and Synchronization of Chua's Circuits", Asian J. of Control, 1-2, pp.75-87, 1999. 
[9] S.S. Ge and C. Wang. "Adaptive Control of Uncertain Chua's Circuit", IEEE Trans. CAS-I, 47-9, pp.1397-1402, 2000.

[10] A.L. Fradkov and A.Y. Markov. "Adaptive Synchronization of Chaotic Systems Based on Speed Gradient Method and Passification", IEEE Trans. CAS-I, 44-10, pp.905-912, 1997.

[11] A. Fradkov, H. Nijmeijer and A. Markov. "Adaptive Observer-Based Synchronization for Communication", Int. J. of Bifurcation and Chaos, 10-12, pp.2807-2813, 2000.

[12] H. Huijberts, H. Nijmeijer and R. Willems. "ASystem Identification in Communication with Chaotic Systems", IEEE Trans. CAS-I, 47-6, pp.800808,2000 .

[13] T. Matsuo and K. Nakano. "Robust stabilization of closed-loop systems by PID+Q controller", Int. J. of Control, 70-4, pp.631-650, 1998.

[14] T. Matsuo, M. Nishikawa, H. Suemitsu and K. Nakano. "Adaptive Decrypter of Chaotic Communication Systems Based on Riccati Equation", Proc. of Asian Control Conference 2002, CD-ROM. pp.99-104, 2002 .

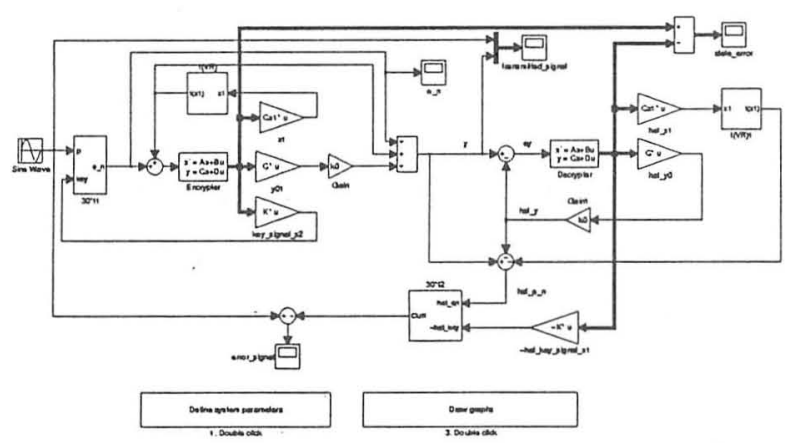

Figure 2: Simulink configuration of cryptosystem proposed by Grassi it et al.

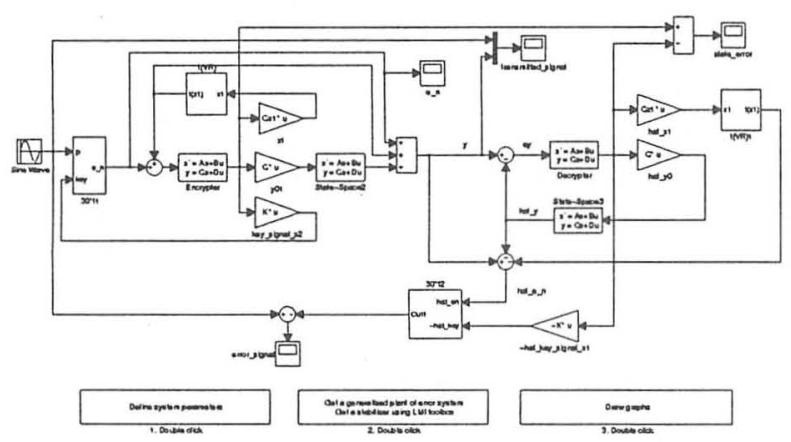

Figure 3: Simulink configuration of cryptosystem proposed here.
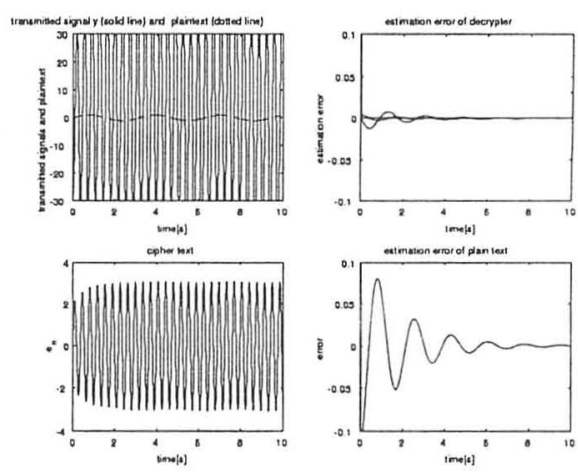

Figure 4: The responses of the cryptosystem proposed by Grassi et al.
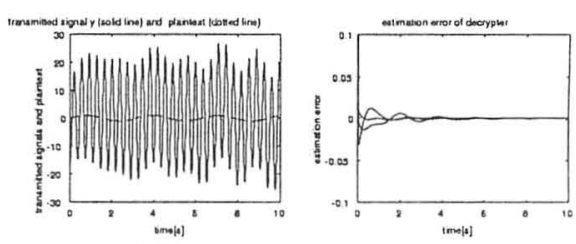

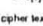
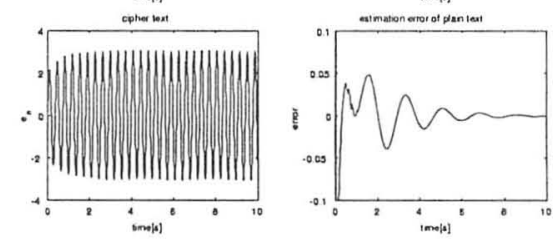

Figure 5: The responses of the cryptosystem proposed here.

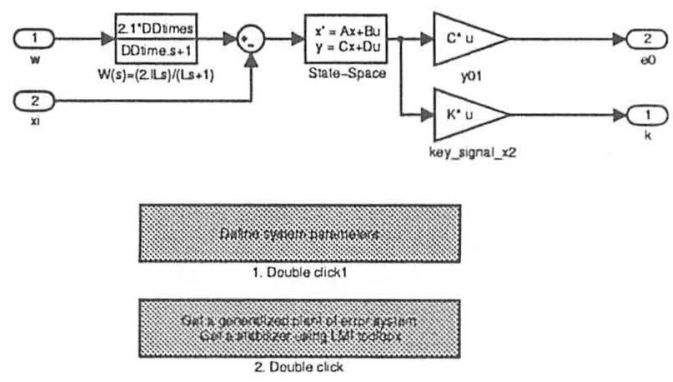

Figure 6: Simulink configuration of generalized plant. 


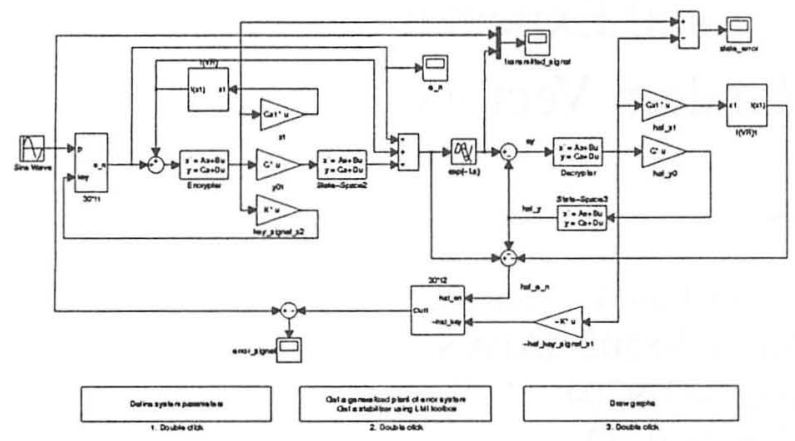

Figure 7: Simulink configuration of cryptosystem proposed here with time delay in transmission line.
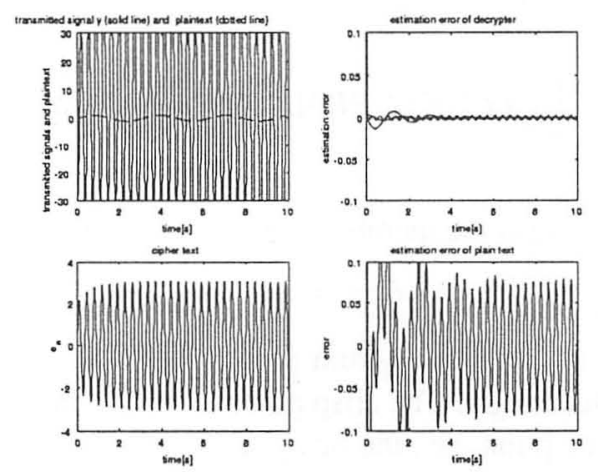

Figure 8: The responses of the cryptosystem proposed by Grassi et al. with time delay in transmission line.
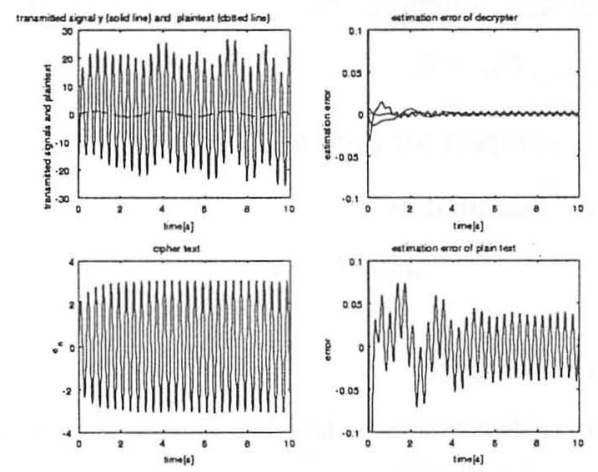

Figure 9: The responses of the cryptosystem proposed here with time delay in transmission line.

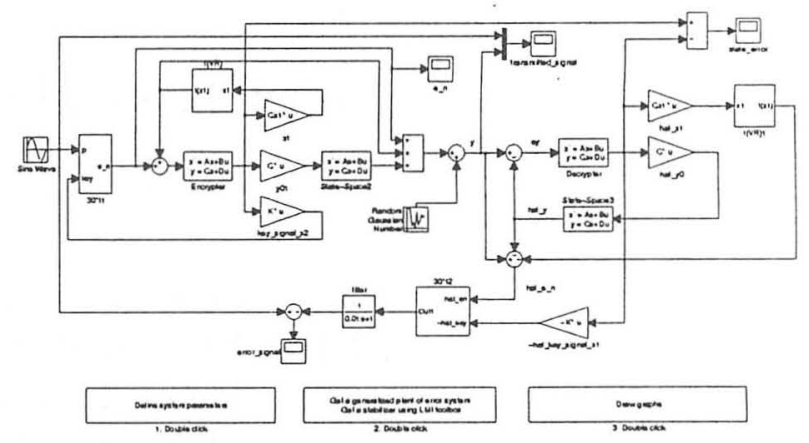

Figure 10: Simulink configuration of cryptosystem proposed here with noise in transmission line.
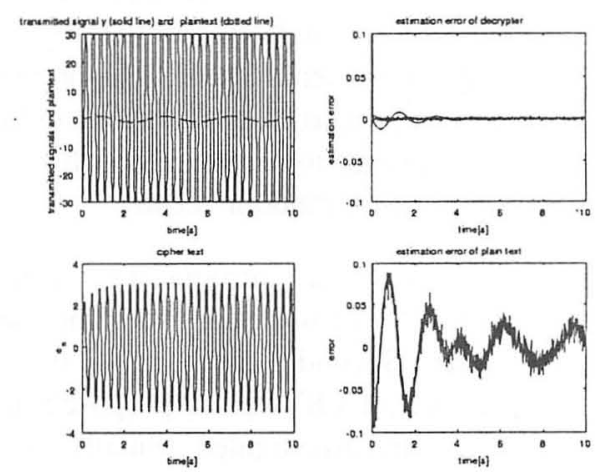

Figure 11: The responses of the cryptosystem proposed by Grassi et al. with noise in transmission line.
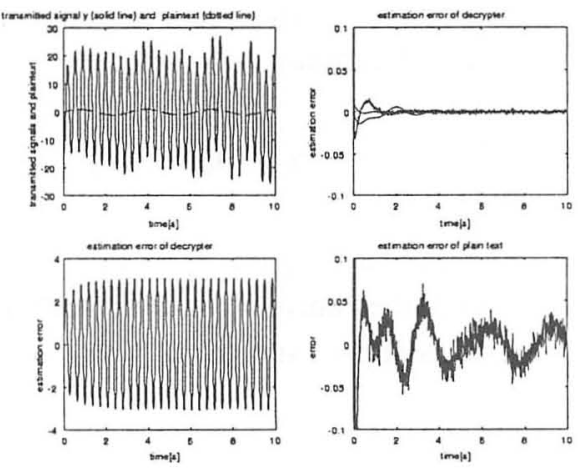

Figure 12: The responses of the cryptosystem proposed here with noise in transmission line. 